\title{
Estudio comparativo de dos complementos alimenticios inhibidores de la $5 \alpha$-reductasa en la alopecia androgénica femenina
}

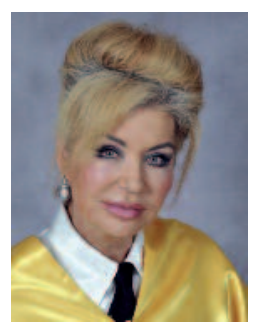

\section{Aurora Guerra-Tapia}

Jefa de la Sección de Dermatología. Hospital Universitario

12 de Octubre. Madrid.

Profesora titular de Dermatología Médico-Quirúrgica y Venereología. Facultad de Medicina. Universidad Complutense de Madrid.

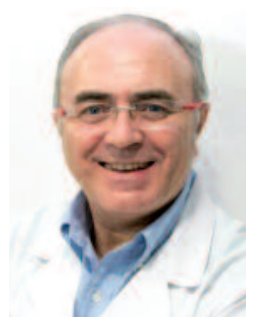

\section{Agustín Buendía Eisman}

Profesor titular de Dermatología. Facultad de Medicina. Universidad de Granada.

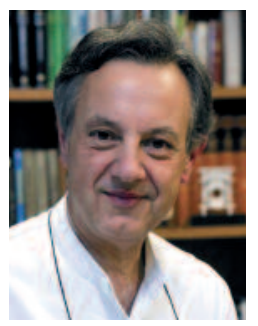

\section{Juan Ferrando Barberá}

Profesor titular de Dermatología. Facultad de Medicina. Universitat de Barcelona. Hospital Clínic de Barcelona.

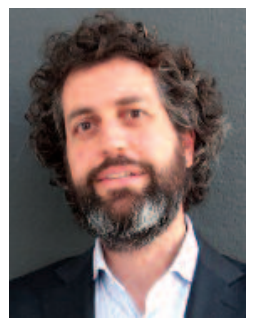

Jordi Picas Jufresa

Responsable de I+D clínica. Laboratorios Reig Jofre. Barcelona.

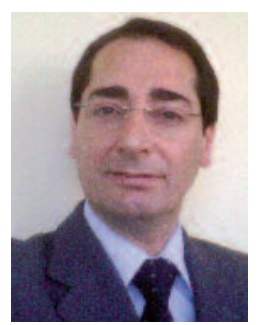

Rafael Bermejo Rodríguez Director médico. Laboratorios Reig Jofre. Madrid.

\section{RESUMEN}

Estudio comparativo controlado con placebo realizado en tres grupos de 20 mujeres cada uno, posmenopáusicas, de edades comprendidas entre los 50 y los 75 años y con alopecia androgénica femenina (AAF) en estadio I-II de la escala de Ludwig.

Los tratamientos asignados según un esquema previo de aleatorización fueron: complemento alimenticio inhibidor de la enzima $5 \alpha$-reductasa con la combinación de los extractos vegetales Pygeum africanum/Serenoa repens en dosis de $100 \mathrm{mg}$ cada uno + metilsulfonilmetano (MSM) en dosis de $500 \mathrm{mg}$; complemento alimenticio inhibidor de la enzima $5 \alpha$-reductasa con la combinación de los extractos vegetales Pygeum africanum/Serenoa repens en dosis de $50 \mathrm{mg}$ cada uno; y placebo.

Se realizaron tres visitas a lo largo del estudio: visita T0 basal a la inclusión en el estudio; visita T8 a las 8 semanas de tratamiento; y visita T16 a las 16 semanas de tratamiento. Se estableció una pauta de tratamiento de 2 cápsulas al día en dos tomas, mañana y noche.

Las variables principales de eficacia evaluadas fueron: fototricograma (cabello total y cabello en fase anágena), resistencia del cabello a la tracción (prueba de tracción o pull test) y evaluación clínica (eritema y prurito). Como variables secundarias, se valoraron: tolerabilidad y cuestionario de evaluación subjetiva del estado del cabello. Todas las variables fueron evaluadas en el momento basal T0, en $\mathrm{T} 8$ a las 8 semanas y en T16 a las 16 semanas de tratamiento, excepto la evaluación subjetiva, que únicamente se realizó en la visita T16.

En el análisis comparativo, se observa a las 16 semanas de tratamiento que, con la formulación Pygeum africanum en dosis de $100 \mathrm{mg} /$ Serenoa repens en dosis de $100 \mathrm{mg}+$ MSM en dosis de $500 \mathrm{mg}$, se obtiene con respecto a la formulación Pygeum africanum en dosis de $50 \mathrm{mg} /$ Serenoa repens en 
dosis de $50 \mathrm{mg}$ : un aumento significativo del número de cabellos totales; un mayor aumento de la proporción de cabellos en fase anágena patente desde la visita T8, siendo un $30 \%$ superior en la visita de las 16 semanas; y una mejora de la resistencia del cabello a la tracción tres veces superior.

A la luz de los resultados obtenidos en el estudio comparativo, se podría concluir que, con las aportaciones de la nueva formulación incrementando la dosis de sus extractos vegetales de 50 a $100 \mathrm{mg}$ e incorporando la nueva molécula de MSM en dosis de $500 \mathrm{mg}$ con propiedades antioxidantes y antiinflamatorias, se objetiva un efecto adicional, «efecto PLUS», que mejora la efectividad del tratamiento y contribuye a un abordaje clínico más integral de la AAF.

Palabras clave: alopecia androgénica, suplemento alimenticio, 5 $\alpha$-reductasa, Pygeum africanum, Serenoa repens, metilsulfonilmetano.

\section{ABSTRACT}

Comparative study controlled with placebo including three groups of 20 50-to-75-yearold postmenopausal women with female androgenetic alopecia (FAA) in stage I-II of the Ludwig scale.

Treatments were assigned according to the previous randomization scheme: a $5 \alpha-$-reductase inhibitor food supplement with the combination of Pygeum africanum/Serenoa repens plant extracts at a dose of $100 \mathrm{mg}$ each one + methylsulfonylmethane (MSM) at a dose of $500 \mathrm{mg}$; a $5 \alpha$-reductase inhibitor food supplement with the combination of Pygeum africanum/Serenoa repens plant extracts at a dose of $50 \mathrm{mg}$ each one; and placebo.

Three visits were made throughout the study: baseline $\mathrm{T} 0$ visit at the inclusion in the study; visit T8 after 8 weeks of treatment; and visit T16 at 16 weeks of treatment. A treatment regimen of 2 capsules per day was established in two doses, in the morning and at night.

The main efficacy variables evaluated were: phototrichogram (total hair and hair in anagen phase), hair resistance to traction (pull test), clinical evaluation (erythema and pruritus). As secondary variables, the following ones were measured: tolerability and a subjective assessment of hair condition questionnaire. All the variables were evaluated at baseline (T0), and at 8 weeks (T8) and 16 weeks (T16) of treatment, except for the subjective evaluation that was only performed at visit T16.

At 16 weeks of treatment, the comparative analysis shows that with the formulation Pygeum africanum at a dose of $100 \mathrm{mg} /$ Serenoa repens at a dose of $100 \mathrm{mg}+\mathrm{MSM}$ at a dose of $500 \mathrm{mg}$ several advantages are obtained over the formulation Pygeum africanum at a dose of $50 \mathrm{mg} /$ Serenoa repens at a dose of $50 \mathrm{mg}$ : a significant increase in the number of total hair; a greater increase in the proportion of hair in the patent anagen phase since the T8 visit, being $30 \%$ higher in the visit at 16 weeks; and a three times better improvement in the resistance of hair to traction.

These findings show us that, in the comparative study, the contributions of the new formulation increasing the dose of its plant extracts from $50 \mathrm{mg}$ to $100 \mathrm{mg}$ and incorporating the new MSM molecule at a dose of $500 \mathrm{mg}$, with antioxidant and anti-inflammatory properties, provides an additional beneficial effect, a «PLUS effect», which improves treatment effectiveness and contributes to a more integrated clinical approach to FAA.

Key words: androgenetic alopecia, food supplement, $5 \alpha$-reductase, Pygeum africanum, Serenoa repens, methylsulfonylmethane. 


\section{INTRODUCCIÓN}

La alopecia androgénica femenina (AAF) es la principal causa de pérdida de pelo en las mujeres adultas, que conlleva un importante impacto psicológico, que afecta a su calidad de vida. Se caracteriza por una miniaturización progresiva de los folículos pilosos, que se produce por una serie de mecanismos fisiopatológicos: acortamiento del ciclo anágeno-telógeno del cabello, pérdida de la estructura y de la densidad capilar, y fibrosis y atrofia del folículo piloso ${ }^{1}$.

Actualmente, se considera que el mecanismo fisiopatológico por el cual tiene lugar la AAF es multifactorial y, en este sentido, además del factor genético y del hormonal, el factor oxidativo y la microinflamación aparecen cada vez con más fuerza como dianas imprescindibles sobre las que se debe intervenir para tratar la $\mathrm{AAF}^{2}$.

Cada uno de estos factores produce una serie de efectos sobre el folículo piloso que contribuyen a la progresión de la AAF, como son:

- Factor hormonal ${ }^{1}$, que, por un exceso de producción de andrógenos y una mayor concentración de la enzima $5 \alpha$-reductasa en los folículos pilosos, va a producir: aumento de la apoptosis de las células de la papila dérmica; aumento de la producción del sebo por la glándula sebácea; reducción de la producción de queratina por los queratinocitos; y reducción del riego sanguíneo y del aporte de oxígeno y nutrientes.

- Factor oxidativo ${ }^{1}$, que, debido a un exceso de especies reactivas del oxígeno y una menor eficiencia en las papilas alopécicas para contrarrestar el estrés oxidativo, va a producir aumento de la apoptosis de las células de la papila dérmica y acortamiento del ciclo anágeno-telógeno.

- Factor infamatorio ${ }^{1}$, que, debido al infiltrado perifolicular de linfocitos, mastocitos y macrófagos, que producen una microinflamación local, va a ocasionar: aumento de la apoptosis de las células de la papila dérmica folicular; aumento de la producción del sebo por la glándula sebá- cea; y depósito anómalo de colágeno con fibrosis y atrofia del folículo.

Ante esta visión multifactorial de la fisiopatología de la AAF, el Departamento de Investigación y Desarrollo de los Laboratorios Reig Jofre se propuso desarrollar una formulación que tratara todos los factores descritos y ofreciera un abordaje clínico más integral de la AAF. Los objetivos que debía cumplir la nueva formulación eran:

1. Incrementar la actividad inhibitoria de la enzima $5 \alpha$-reductasa de los extractos que han mostrado el mejor nivel de eficacia y seguridad a lo largo de su experiencia clínica.

2. Incorporar una nueva molécula que aportara un valor añadido al tratamiento de la AAF por sus efectos antioxidantes y antiinflamatorios, que vendría a contribuir a ese abordaje clínico más integral que se pretende.

Finalmente, en la composición de la nueva formulación, encontraremos dosis de Serenoa repens y Pygeum africanum aumentada a $100 \mathrm{mg}$ para cada uno de los extractos, y la incorporación de metilsulfonilmetano (MSM) en dosis de $500 \mathrm{mg}$, que aporta actividad antioxidante y antiinflamatoria.

Con el fin de objetivar los beneficios clínicos de la nueva formulación, se realizó un estudio comparativo de los complementos alimenticios Complidermol $5 \alpha$ y Complidermol $5 \alpha$ PLUS, cuyas principales diferencias en su composición son: la dosis de los extractos vegetales Serenoa repensPygeum africanum (50 mg de cada uno frente a la dosificación de $100 \mathrm{mg}$ cada uno, respectivamente); y la incorporación en el segundo de la molécula MSM en dosis de $500 \mathrm{mg}$, con efecto antioxidante y antiinflamatorio. A continuación, pasamos a describir los objetivos, el diseño y los resultados de este estudio.

\section{OBJETIVO}

El objetivo principal del estudio fue evidenciar y cuantificar el efecto beneficioso adicional («efec- 
to PLUS») en cuanto a la efectividad del tratamiento (número de cabellos totales, proporción de cabellos en fase anágena y prueba de tracción) que aporta la formulación del complemento alimenticio con Serenoa repens-Pygeum africanum en dosis de $100 \mathrm{mg}$ y MSM en dosis de $500 \mathrm{mg}$ frente a la formulación del complemento alimenticio con Serenoa repens-Pygeum africanum en dosis de $50 \mathrm{mg}$.

Como objetivos secundarios, se quiso cuantificar la tolerabilidad de ambas formulaciones, así como valorar la satisfacción global de las pacientes con la nueva formulación.

\section{DISEÑO}

Estudio comparativo, observacional, prospectivo, aleatorizado y controlado con placebo, realizado en tres grupos de 20 mujeres posmenopáusicas cada uno con AAF, a las que se les asignó el tratamiento por orden de presentación en el centro del estudio según un esquema de aleatorización previo.

Los tratamientos asignados fueron: complemento alimenticio inhibidor de la enzima $5 \alpha-$ reductasa con la combinación de los extractos vegetales Pygeum africanum/Serenoa repens en dosis de $100 \mathrm{mg}$ cada uno + MSM en dosis de $500 \mathrm{mg}$; complemento alimenticio inhibidor de la enzima $5 \alpha$-reductasa con la combinación de los extractos vegetales Pygeum africanum/Serenoa repens en dosis de $50 \mathrm{mg}$ cada uno; y placebo.

Se establecieron tres visitas a lo largo del estudio: visita T0 basal a la inclusión del caso en el estudio; visita T8 a las 8 semanas de tramiento; $y$ visita T16 a las 16 semanas de tratamiento. Durante el tiempo de seguimiento, se fijó una pauta de tratamiento, de acuerdo con las recomendaciones de empleo establecidas para este tipo de preparados en el tratamiento de la AAF, de 2 cápsulas al día en dos tomas, una por la mañana y otra por la noche.

\section{METODOLOGÍA}

El estudio se llevó a cabo de conformidad con los requisitos del sistema de garantía de calidad, de acuerdo con el principio de buenas prácticas de laboratorio y buenas prácticas clínicas, así como los principios establecidos por la Asociación Médica Mundial en la Declaración de Helsinki.

Se realizó con la colaboración del Instituto de Investigación de la Piel y Evaluación del Producto (ISPE) de Milán. Se inició en enero de 2017, con la inclusión de los datos del primer caso, y se terminó de incorporar los datos del último caso en septiembre de 2017.

Todas las participantes otorgaron su consentimiento informado por escrito antes de iniciar su participación en el estudio.

\section{Perfil de casos}

\section{Criterios de inclusión}

En el estudio, participaron mujeres posmenopáusicas de edades comprendidas entre los 50 y los 75 años, con alopecia androgénica en estadio I-II de la escala de Ludwig. Todas ellas debían ser capaces de seguir las directrices del estudio y cumplir con todas las visitas de seguimiento.

\section{Criterios de exclusión}

Ninguna de las participantes debía presentar antecedentes de reacciones cutáneas inusuales a productos de higiene facial, cosméticos, o sensibilidad a cualquiera de los componentes de los productos en estudio; no debían tomar fármacos tópicos o sistémicos que pudieran afectar a los resultados del análisis (antiinflamatorios, corticosteroides, etc.); no debían sufrir enfermedades sistémicas o alteraciones cutáneas (como eccema, psoriasis, acné grave, etc.) que pudieran interferir en la evaluación de los productos en estudio o que aumentaran el riesgo para ellas; no debían haber utilizado tratamientos para evitar la caída del cabello (tópicos o sistémicos) en los tres meses previos a la entrada en este estudio, y no debían haber participado en otra investigación clínica con objetivos comparables en los tres meses previos a la entrada en este estudio. 


\section{Variables de eficacia}

Se realizaron las siguientes evaluaciones como variables principales: fototricograma, resistencia del cabello a la tracción (prueba de tracción o pull test) y evaluación clínica (síntomas: eritema y prurito). Como variables secundarias, se evaluaron la tolerabilidad y la evaluación subjetiva del estado del cabello.

Todas las variables fueron evaluadas en el momento basal T0, en T8 a las 8 semanas y en T16 a las 16 semanas de tratamiento, excepto la evaluación subjetiva, que únicamente se realizó a las 16 semanas.

\section{Fototricograma}

El análisis del fototricograma se realizó utilizando el sistema TrichoScan ${ }^{\circledR}$, basado en el principio de la microscopia epiluminiscente e incluyendo el Fotofinder Dermoscope ${ }^{\circledR}$ con el programa TrichoScan Professional v2. $0^{\circledR}$, para evaluar el número total y porcentaje de cabellos en fase anágena (fase de crecimiento activo) y telógena (fase de reposo) en un área de $0,728 \mathrm{~cm}^{2}$. Este análisis buscaba, fundamentalmente, el aumento del porcentaje de cabellos en fase anágena.

\section{Prueba de tracción}

La evaluación de la resistencia del cabello a la tracción o prueba de la tracción se realizó aplicando una tracción constante en un mechón de cabello en tres zonas distintas del cuero cabelludo (temporal, frontal y occipital). Se evaluó en función del número total de cabellos eliminados en las tres zonas, utilizando una escala de 4 puntos, donde $0=>6$ cabellos, $1=6-4$ cabellos, $2=3-1$ cabellos, y 3 = 0 cabellos.

\section{Evaluación clínica}

La evaluación clínica se centró en el análisis en el grupo Pygeum africanum/Serenoa repens en dosis de $100 \mathrm{mg}$ y MSM en dosis de $500 \mathrm{mg}$ y en la evaluación de aquellos síntomas objetivables más relacionados con el componente inflamatorio de la AAF, seleccionando entre los posibles los síntomas eritema y prurito. Para su evaluación, se generó una escala de 0 a 3 , donde $0=$ ausencia, 1 = leve, 2 = moderado, y 3 = grave. También se evaluó la tolerabilidad global del tratamiento en una escala de 4 puntos, donde $0=$ ausencia (requiriendo el abandono del tratamiento), $1=$ mala (no requiriendo el abandono del tratamiento, pero presentando efectos adversos moderados), 2 = moderada (con efectos adversos leves y transitorios), y 3 =óptima/buena (sin efectos adversos).

\section{Evaluación subjetiva}

Para la evaluación subjetiva, las participantes expresaron su opinión sobre la eficacia y la aceptabilidad del producto mediante un cuestionario que preguntaba acerca de su satisfacción respecto de: la disminución de la pérdida de pelo; el aumento de la densidad de pelo; pelo más fuerte; y pelo más sano. El resultado se expresaba en porcentaje del grado de satisfacción medido en una escala de 4 puntos para cada parámetro, donde se consideraba 1 = nada efectivo, 2 = poco efectivo, $3=$ bastante efectivo y 4 = muy efectivo.

Se realizaron análisis descriptivos para todas las variables registradas. Las variables categóricas se presentaron como frecuencia y porcentajes, mientras que las variables continuas se presentaron como número de participantes, media y desviación estándar. Se utilizaron las pruebas de Kolmogórov-Smirnov para los análisis de normalidad de los resultados, y se compararon los datos obtenidos en las tres visitas (basal, 8 semanas y 16 semanas) utilizando el análisis Anova de Friedman y el coeficiente de concordancia de Kendall para los datos no paramétricos dependientes. Las comparaciones de los datos obtenidos en cada visita entre tratamientos se realizaron utilizando la prueba de la $U$ de Mann-Whitney para datos no paramétricos independientes. Se consideró estadísticamente significativo un valor de significación estadística $[p]<0,05$. 


\section{RESULTADOS}

De las 20 mujeres incluidas en cada grupo, dos del grupo que recibieron Pygeum africanum/Serenoa repens en dosis de $50 \mathrm{mg}$ abandonaron el estudio (una de ellas por reacción alérgica desarrollada a alguno de los componentes del preparado a las 4 semanas de tratamiento y la otra por motivos ajenos a la administración del preparado). En el grupo que recibió placebo, también abandonaron el estudio dos participantes (ambas por motivos no relacionados con la administración del preparado). En el grupo que recibió Pygeum africanum/Serenoa repens en dosis de $100 \mathrm{mg}$ con MSM en dosis de $500 \mathrm{mg}$, no hubo ningún abandono.

Solo las participantes que completaron el estudio fueron incluidas en los análisis de eficacia y tolerabilidad. En la tabla 1, se muestra la compilación de los datos estadísticos de las variables principales en cada una de las visitas a lo largo de todo el estudio.

\section{Fototricograma}

El número total de cabellos aumentó significativamente en el grupo de Pygeum africanum/Serenoa repens en dosis de $100 \mathrm{mg}$ con MSM en dosis de $500 \mathrm{mg}$ tras 8 semanas de tratamiento (variación T8-T0: $1,8[1,1 \%], p<0,05)$ y tras 16 semanas (variación T16-T0: 4,0 [2,4\%], $p<0,0001$ ) (tabla 1).

En el análisis comparativo, resulta un incremento en el número total de cabellos superior en el grupo que recibió el preparado con Pygeum africanum/Serenoa repens en dosis de $100 \mathrm{mg}$ y MSM en dosis de $500 \mathrm{mg}$ con respecto al grupo que recibió el preparado con Pygeum africanum/Serenoa repens en dosis de $50 \mathrm{mg}$.

En cuanto a la comparación entre grupos, se encontraron diferencias significativas en la variación del número total de cabellos entre Pygeum africanum/Serenoa repens en dosis de $100 \mathrm{mg}$ con MSM en dosis de $500 \mathrm{mg}$ y el placebo a las 16 semanas $(p<0,0001)$ (tabla 1$)$.

El porcentaje de cabellos en fase anágena aumentó significativamente a las 8 y 16 semanas de tratamiento con Pygeum africanum/Serenoa repens en dosis de $100 \mathrm{mg}$ con MSM en dosis de $500 \mathrm{mg}$ (los incrementos o variaciones obtenidas fueron: $\Delta \mathrm{T} 8$ T0: 3,9 [6,5\%], $p<0,0001 ; \Delta \mathrm{T} 16-\mathrm{T} 0: 7,6$ [12,7\%], $p<0,0001)$ y con Pygeum africanum/Serenoa repens en dosis de $50 \mathrm{mg}(\Delta \mathrm{T} 8$-T0: 3,8 [5,8\%], $p<0,05$; $\Delta \mathrm{T} 16-\mathrm{T} 0: 6,3[9,6 \%], p<0,0001)$. En la figura 1, se expresa como el porcentaje del número de cabellos en fase anágena en cada una de las visitas y, en la figura 2, se expresa como el incremento en porcentaje del valor anterior de una visita a otra, desde la visita basal T0 hasta la visita T16 a las 16 semanas de tratamiento.

En el análisis comparativo que se observa en la figura 2, resulta un incremento en el número total de cabellos en fase anágena a las 16 semanas de tratamiento más de un $30 \%$ superior en el grupo que recibió el preparado con Pygeum africanum/Serenoa repens en dosis de $100 \mathrm{mg}$ y MSM en dosis de $500 \mathrm{mg}$ que en el grupo que recibió el preparado con Pygeum africanum/Serenoa repens en dosis de $50 \mathrm{mg}$.

La diferencia en el incremento de cabellos en fase anágena también fue significativa en la comparación entre Pygeum africanum/Serenoa repens en dosis de $100 \mathrm{mg}$ con MSM en dosis de $500 \mathrm{mg}$ y placebo a las 8 semanas $(p<0,01)$ y a las 16 semanas $(p<0,0001)$ (tabla 1$)$.

Por otro lado, el preparado de Pygeum africanum/Serenoa repens en dosis de $100 \mathrm{mg}$ con MSM en dosis de $500 \mathrm{mg}$ presentó una mayor proporción de pacientes con mejoría en el porcentaje de cabellos en fase anágena que el preparado de Pygeum africanum/Serenoa repens en dosis de $50 \mathrm{mg}$ a las 8 semanas (del $95 \%$ frente al $83 \%$, respectivamente) y a las 16 semanas (del $100 \%$ frente al $83 \%$, respectivamente). Ambos tratamientos presentaron un mayor porcentaje de pacientes con mejoría a las 8 y 16 semanas que con el placebo (del 39 y del $50 \%$, respectivamente, para el placebo) (tabla 2).

\section{Pueba de tracción}

En los resultados de la prueba de tracción, se observó una mejora significativa tanto de su valor numé- 
Guerra-Tapia A et al. Estudio comparativo de dos complementos alimenticios inhibidores de la $5 \alpha$-reductasa en la alopecia androgénica femenina

Tabla 1. Compilación de datos del resumen de los resultados

\begin{tabular}{l|c|c|c|c|c|c|c}
\hline Tratamiento & $\begin{array}{c}\text { T0 } \\
\text { Media } \pm \text { DE }\end{array}$ & $\begin{array}{c}\text { T8 } \\
\text { Media } \pm \text { DE }\end{array}$ & $\begin{array}{c}\text { T16 } \\
\text { Media } \pm \text { DE }\end{array}$ & $\begin{array}{c}\text { Variación } \\
\text { T8-T0 }\end{array}$ & $\begin{array}{c}\text { Variación } \\
\text { T16-T0 }\end{array}$ & $\begin{array}{c}\text { T0 vs. T8 } \\
\text { valor de } p\end{array}$ & $\begin{array}{c}\text { T0 vs. T16 } \\
\text { valor de } p\end{array}$ \\
\hline
\end{tabular}

Número total de cabellos (n. $\left.{ }^{\circ}\right)$

\begin{tabular}{l|l|l|l|l|l|l|l}
\hline $\begin{array}{l}\text { Pygeum africanum- } \\
\text { Serenoa repens en } \\
\text { dosis de } 100 \mathrm{mg} \\
+\begin{array}{l}\text { MSM en dosis } \\
\text { de } 500 \mathrm{mg}\end{array}\end{array}$ & $166,4 \pm 35,7$ & $168,2 \pm 36,4$ & $170,4 \pm 36,9$ & $\begin{array}{c}1,8 \\
(1,1 \%)\end{array}$ & $\begin{array}{c}4,0 * \\
(2,4 \%)\end{array}$ & $<\mathbf{0 , 0 5}$ & $<\mathbf{0 0 0 1}$ \\
\hline $\begin{array}{l}\text { Pygeum africanum- } \\
\begin{array}{l}\text { Serenoa repens en } \\
\text { dosis de } 50 \mathrm{mg}\end{array}\end{array}$ & $152,2 \pm 48,3$ & $154,4 \pm 48,8$ & $152,2 \pm 45,0$ & $\begin{array}{c}2,2 \\
(1,4 \%)\end{array}$ & $\begin{array}{c}0 \\
(0 \%)\end{array}$ & $>0,05$ & $>0,05$ \\
\hline \begin{tabular}{l} 
Placebo \\
\hline
\end{tabular} & $203,7 \pm 54,5$ & $202,9 \pm 57,5$ & $202,2 \pm 54,1$ & $\begin{array}{c}-0,8 \\
(-0,4 \%)\end{array}$ & $\begin{array}{c}-1,5 \\
(-0,7 \%)\end{array}$ & $>0,05$ & $>0,05$ \\
\hline
\end{tabular}

Porcentaje de cabello en fase anágena (\%)

\begin{tabular}{|c|c|c|c|c|c|c|c|}
\hline $\begin{array}{l}\text { Pygeum africanum- } \\
\text { Serenoa repens en } \\
\text { dosis de } 100 \mathrm{mg} \\
+ \text { MSM en dosis } \\
\text { de } 500 \mathrm{mg}\end{array}$ & $60,1 \pm 8,5$ & $64,0 \pm 8,4$ & $67,7 \pm 8,2$ & $\begin{array}{c}3,9 * * \\
(6,5 \%)\end{array}$ & $\begin{array}{c}7,6 * * * \\
(12,7 \%)\end{array}$ & $<0,0001$ & $<0,0001$ \\
\hline $\begin{array}{l}\text { Pygeum africanum- } \\
\text { Serenoa repens en } \\
\text { dosis de } 50 \mathrm{mg}\end{array}$ & $65,9 \pm 10,0$ & $69,7 \pm 10,0$ & $72,2 \pm 7,9$ & $\begin{array}{c}3,8 \\
(5,8 \%)\end{array}$ & $\begin{array}{c}6,3 \\
(9,6 \%)\end{array}$ & $<0,05$ & $<0,0001$ \\
\hline Placebo & $61,4 \pm 8,9$ & $61,2 \pm 9,0$ & $61,0 \pm 9,0$ & $\begin{array}{c}-0,2 \\
(-0,3 \%)\end{array}$ & $\begin{array}{c}-0,4 \\
(-0,4 \%)\end{array}$ & $>0,05$ & $>0,05$ \\
\hline
\end{tabular}

Prueba de tracción (escala de 4 puntos)

\begin{tabular}{|c|c|c|c|c|c|c|c|}
\hline $\begin{array}{l}\text { Pygeum africanum- } \\
\text { Serenoa repens en } \\
\text { dosis de } 100 \mathrm{mg} \\
+\mathrm{MSM} \text { en dosis } \\
\text { de } 500 \mathrm{mg}\end{array}$ & $0,7 \pm 0,5$ & $1,1 \pm 0,5$ & $1,6 \pm 0,5$ & $\begin{array}{c}0,4 \\
(57,1 \%)\end{array}$ & $\begin{array}{c}0,9 * * \\
(128,6 \%)\end{array}$ & $<0,01$ & $<0,0001$ \\
\hline $\begin{array}{l}\text { Pygeum africanum- } \\
\text { Serenoa repens en } \\
\text { dosis de } 50 \text { mg }\end{array}$ & $1,7 \pm 0,5$ & $2,1 \pm 0,6$ & $2,4 \pm 0,6$ & $\begin{array}{c}0,4 \\
(23,5 \%)\end{array}$ & $\begin{array}{c}0,7 \\
(41,2 \%)\end{array}$ & $<0,05$ & $<0,01$ \\
\hline Placebo & $1,7 \pm 0,7$ & $1,8 \pm 0,8$ & $1,8 \pm 0,7$ & $\begin{array}{c}0,1 \\
(5,9 \%)\end{array}$ & $\begin{array}{c}0,1 \\
(5,9 \%)\end{array}$ & $>0,05$ & $>0,05$ \\
\hline
\end{tabular}

DE: desviación estándar; MSM: metilsulfonilmetano; $p$ : nivel de significación estadística; T0: visita a las 0 semanas de tratamiento (basal, a la inclusión en el estudio); T8: visita a las 8 semanas de tratamiento; T16: visita a las 16 semanas de tratamiento; vs.: versus.

*Pygeum africanum/Serenoa repens en dosis de $100 \mathrm{mg}$ con MSM en dosis de 500 mg vs. placebo: $p$ < 001 . **Pygeum africanum/Serenoa repens en dosis de $100 \mathrm{mg}$ con MSM en dosis de $500 \mathrm{mg}$ vs. placebo: $p$ <,01.

$* * *$ Pygeum africanum/Serenoa repens en dosis de $100 \mathrm{mg}$ con MSM en dosis de $500 \mathrm{mg}$ vs. placebo: $p<0,0001$. 
Estudio comparativo de dos complementos alimenticios inhibidores de la $5 \alpha$-reductasa en la alopecia androgénica femenina

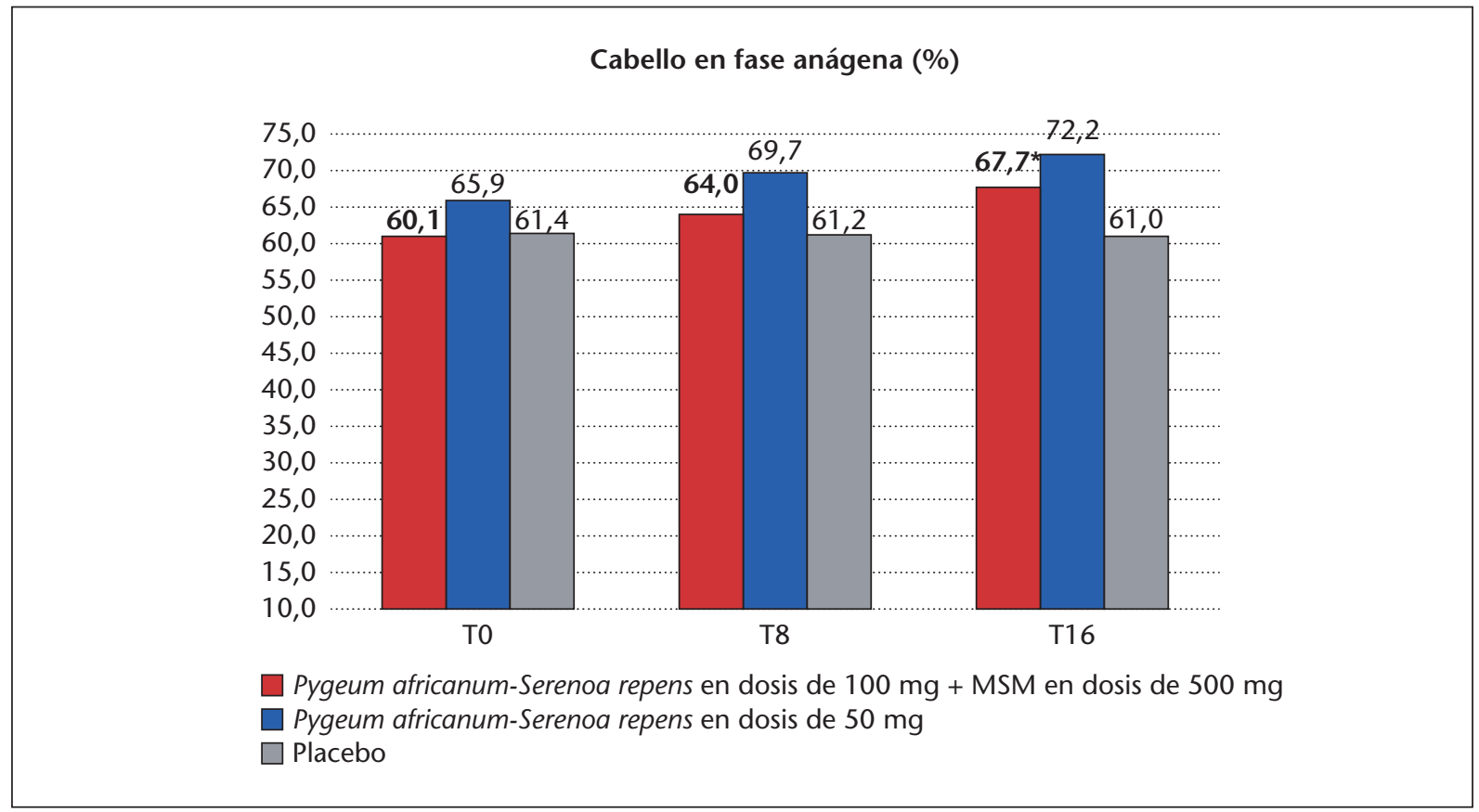

Figura 1. Cabello en fase anágena. Proporción en porcentaje (\%).

MSM: metilsulfonilmetano; T0: visita a las 0 semanas de tratamiento (basal, a la inclusión en el estudio); T8: visita a las 8 semanas de tratamiento; T16: visita a las 16 semanas de tratamiento.

*significación: véase la tabla compilada.

\section{Incremento de cabello en fase anágena (\%)}

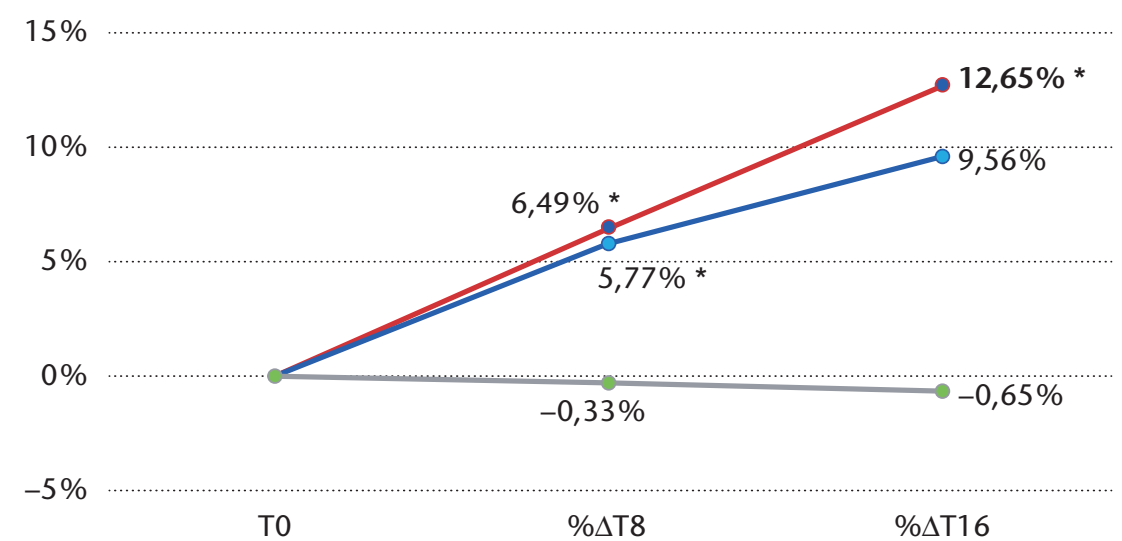
$\rightarrow$ Pygeum africanum-Serenoa repens en dosis de $100 \mathrm{mg}+\mathrm{MSM}$ en dosis de $500 \mathrm{mg}$
$\rightarrow$ Pygeum africanum-Serenoa repens en dosis de $50 \mathrm{mg}$ - Placebo

Figura 2. Cabello en fase anágena. Incremento en porcentaje (\%) respecto de la visita basal.

$\Delta$ : incremento; MSM: metilsulfonilmetano; T0: visita a las 0 semanas de tratamiento (basal, a la inclusión en el estudio); T8: visita a las 8 semanas de tratamiento; T16: visita a las 16 semanas de tratamiento.

*significación: véase la tabla compilada. 
Guerra-Tapia A et al. Estudio comparativo de dos complementos alimenticios inhibidores de la $5 \alpha$-reductasa en la alopecia androgénica femenina

Tabla 2. Porcentaje de casos con mejora de cabellos en fase anágena

\begin{tabular}{l|c|c}
\hline Grupo & T8-T0 & T16-T0 \\
\hline $\begin{array}{l}\text { Pygeum africanum/Serenoa repens en dosis de } 100 \mathrm{mg} \text { con } \\
\text { MSM en dosis de } 500 \mathrm{mg}\end{array}$ & $18(95 \%)$ & $20(100 \%)$ \\
\hline Pygeum africanum/Serenoa repens en dosis de $50 \mathrm{mg}$ & $15(83 \%)$ & $15(83 \%)$ \\
\hline Placebo & $7(39 \%)$ & $9(50 \%)$ \\
\hline
\end{tabular}

MSM: metilsulfonilmetano; T0: visita a las 0 semanas de tratamiento (basal, a la inclusión en el estudio); T8: visita a las 8 semanas de tratamiento; T16: visita a las 16 semanas de tratamiento.

rico en la escala de $0-3$ como en su porcentaje de mejora respecto de la visita basal en los dos grupos activos. No se observó aumento significativo en el grupo del placebo. Los valores fueron a las 8 y 16 semanas de tratamiento: con Pygeum africanum/Serenoa repens en dosis de $100 \mathrm{mg}$ con MSM en dosis de $500 \mathrm{mg}: \Delta \mathrm{T} 8$-T0: $0,4(57,1 \%), p<0,01$; $\Delta$ T16-T0: 0,9 (128,6\%), $p<0,0001 ;$ y con Pygeum africanum/Serenoa repens en dosis de $50 \mathrm{mg}: \Delta \mathrm{T} 8$ -
T0: $0,4(23,5 \%), p<0,05 ; \Delta \mathrm{T} 16-\mathrm{T} 0: 0,7(41,2 \%)$, $p<0,01$. En la figura 3 , se expresa en el valor numérico de la escala en cada una de las visitas y, en la figura 4, se expresa como el incremento en porcentaje del valor anterior desde la visita basal T0 hasta la vista T16 a las 16 semanas de tratamiento. En el análisis comparativo que se observa en la figura 4, resulta un incremento en resistencia del cabello a la tracción más de tres veces superior

Prueba de tracción (valor en una escala de 0-3)

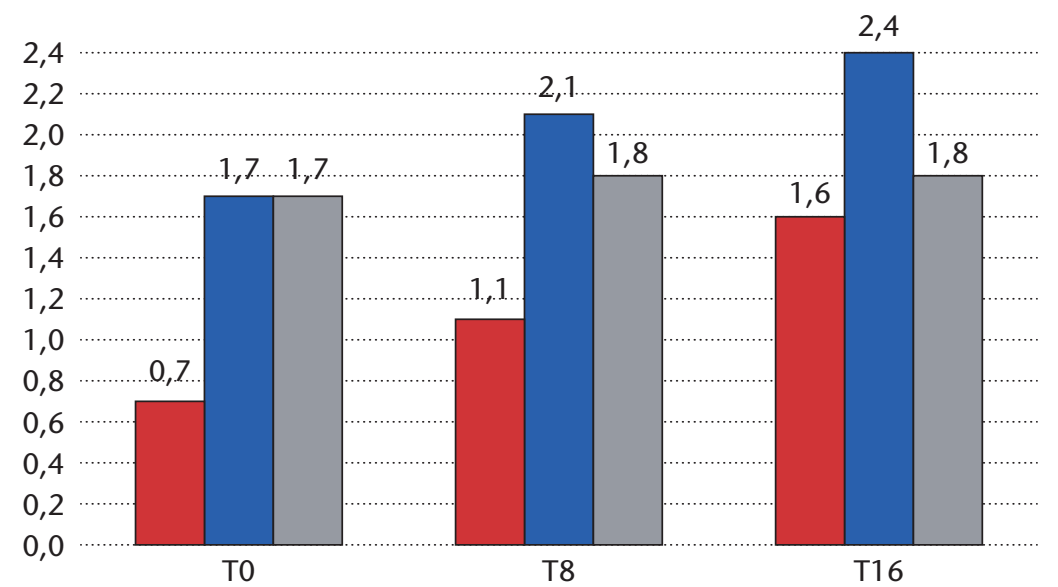

Pygeum africanum-Serenoa repens en dosis de $100 \mathrm{mg}+\mathrm{MSM}$ en dosis de $500 \mathrm{mg}$

Pygeum africanum-Serenoa repens en dosis de $50 \mathrm{mg}$

Placebo

Figura 3. Prueba de tracción. Valor en la escala de 0-3.

MSM: metilsulfonilmetano; T0: visita a las 0 semanas de tratamiento (basal, a la inclusión en el estudio); T8: visita a las 8 semanas de tratamiento; T16: visita a las 16 semanas de tratamiento. 


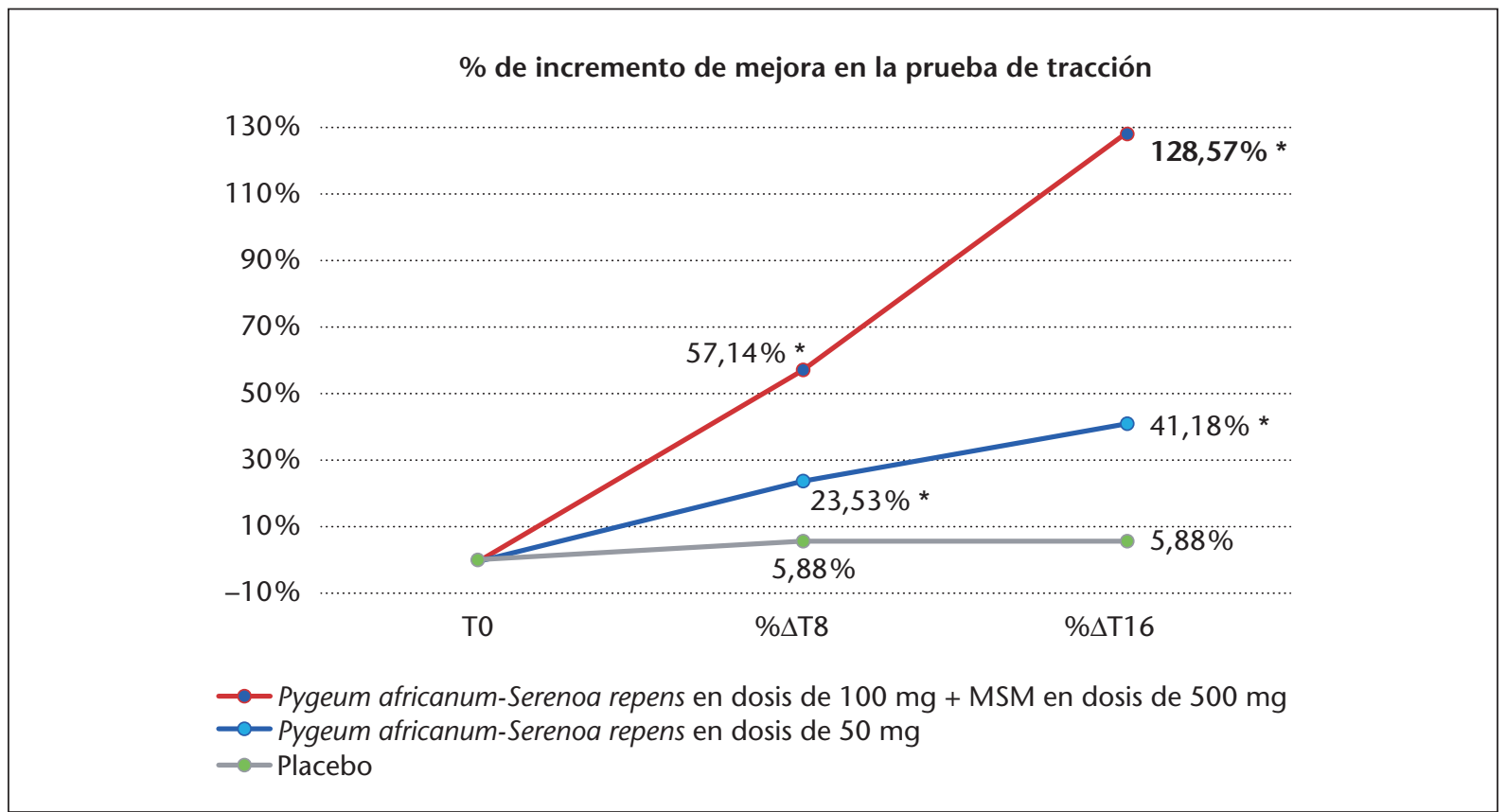

Figura 4. Prueba de tracción. Incremento de mejora en porcentaje (\%) respecto de la visita basal.

$\Delta$ : incremento; MSM: metilsulfonilmetano; T0: visita a las 0 semanas de tratamiento (basal, a la inclusión en el estudio); T8: visita a las 8 semanas de tratamiento; T16: visita a las 16 semanas de tratamiento.

*significación: véase la tabla compilada.

en el grupo que recibió el preparado con Pygeum africanum/Serenoa repens en dosis de $100 \mathrm{mg}$ y MSM en dosis de $500 \mathrm{mg}$ que en el grupo que recibió el preparado con Pygeum africanum/Serenoa repens en dosis de $50 \mathrm{mg}$.

La diferencia en el incremento en la escala de 0-3 en la prueba de tracción también fue significativa en la comparación entre Pygeum africanum/Serenoa repens en dosis de $100 \mathrm{mg}$ con MSM en dosis de $500 \mathrm{mg}$ y el placebo a las 8 semanas $(p<0,01)$ y a las 16 semanas $(p<0,0001)$.

Por otra parte, el preparado de Pygeum africanum/Serenoa repens en dosis de $100 \mathrm{mg}$ con MSM en dosis de $500 \mathrm{mg}$ presentó un mayor porcentaje de pacientes con mejoría en la prueba de tracción que el preparado de Pygeum africanum/Serenoa repens en dosis de $50 \mathrm{mg}$ a las 16 semanas (del $85 \%$ frente al $61 \%$, respectivamente). Ambos tratamientos presentaron un mayor porcentaje de pacientes con mejoría a las 16 semanas que con el placebo (17\%) (tabla 3).

\section{Evaluación clínica}

La evaluación de la tolerabilidad fue óptima/buena tanto a las 8 como a las 16 semanas de tratamiento en todos los casos y grupos de tratamiento. Se observó una puntuación de valor 3 , que se mantuvo tanto en la visita T8 como en la T16. Por otro lado, se observó una disminución significativa $(p<0,05)$ tanto del eritema a las 16 semanas como del prurito a las 8 y 16 semanas con Pygeum africanum/Serenoa repens en dosis de $100 \mathrm{mg}$ con MSM en dosis de $500 \mathrm{mg}$ (fig. 5).

\section{Evaluación subjetiva}

La evaluación subjetiva mostró porcentajes de satisfacción elevados tras 16 semanas de tratamiento con Pygeum africanum/Serenoa repens en dosis de $100 \mathrm{mg}$ con MSM en dosis de $500 \mathrm{mg}$ en todos los parámetros evaluados, siendo el porcentaje de pacientes que consideraron el tratamiento muy efectivo o bastante efectivo: disminución de la pér- 
Guerra-Tapia A et al. Estudio comparativo de dos complementos alimenticios inhibidores de la $5 \alpha$-reductasa en la alopecia androgénica femenina

Tabla 3. Porcentaje de casos con mejora en la prueba de tracción

\begin{tabular}{|c|c|c|}
\hline Grupo & T8-т0 & T16-T0 \\
\hline $\begin{array}{l}\text { Pygeum africanum/Serenoa repens en dosis de } 100 \mathrm{mg} \text { con } \\
\text { MSM en dosis de } 500 \mathrm{mg}\end{array}$ & $7(35 \%)$ & $17(85 \%)$ \\
\hline Pygeum africanum/Serenoa repens en dosis de $50 \mathrm{mg}$ & $7(39 \%)$ & $11(61 \%)$ \\
\hline Placebo & $3(17 \%)$ & $3(17 \%)$ \\
\hline
\end{tabular}

MSM: metilsulfonilmetano; T0: visita a las 0 semanas de tratamiento (basal, a la inclusión en el estudio); T8: visita a las 8 semanas de tratamiento; T16: visita a las 16 semanas de tratamiento.

dida de cabello (90\%), incremento de la densidad del cabello (80\%), cabello más fuerte $(85 \%)$ y cabello más sano (90\%) (fig. 6).

\section{DISCUSIÓN}

Serenoa repens es el extracto con mayor evidencia científica acerca de su actividad inhibidora de la enzima $5 \alpha$-reductasa, seguido por el extracto de
Pygeum africanum. Además, ambos extractos pueden usarse en asociación para incrementar sus efectos, actuando de manera sinérgica en la inhibición de la enzima $5 \alpha$-reductasa ${ }^{3-5}$.

En lo referente a la seguridad de los extractos de Serenoa repens y Pygeum africanum, estos han sido considerados por la Organización Mundial de la Salud (OMS), en su monografía WHO monographs on selected medicinal plants volume 2, como bien tole-

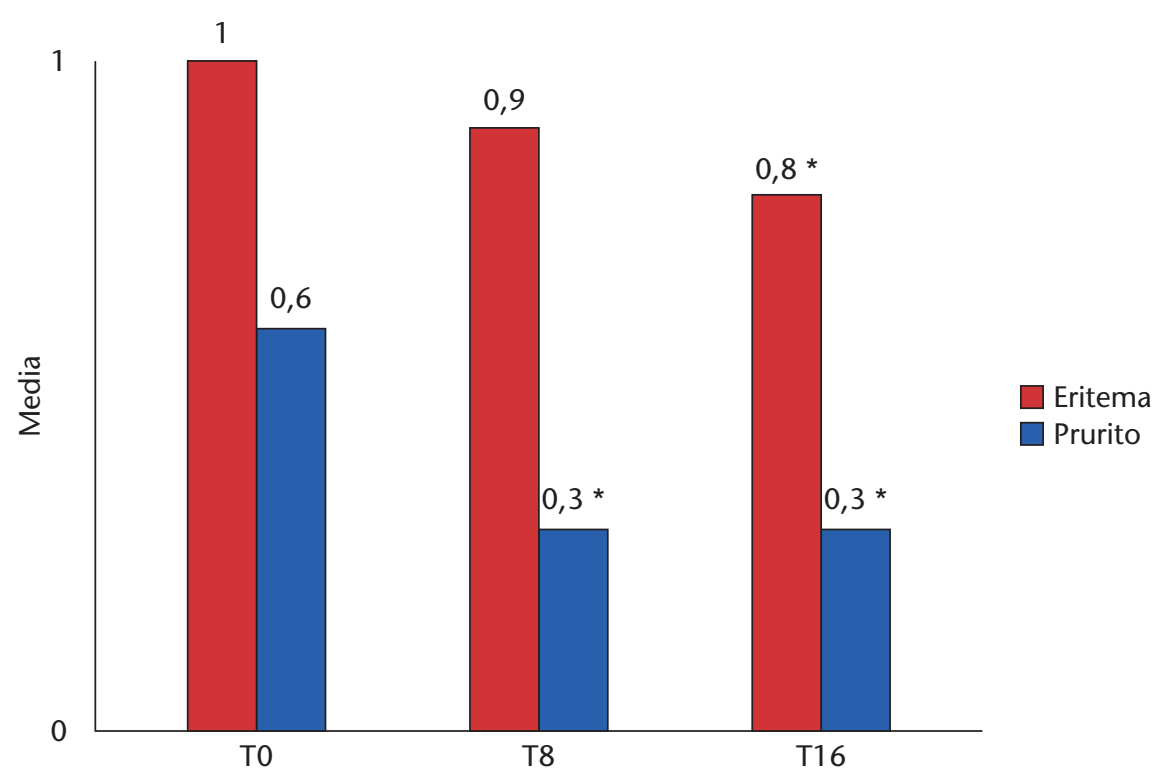

Figura 5. Evaluación clínica. Sintomatología. Pygeum africanum-Serenoa repens en dosis de $100 \mathrm{mg}$ con MSM en dosis de $500 \mathrm{mg}$. MSM: metilsulfonilmetano; T0: visita a las 0 semanas de tratamiento (basal, a la inclusión en el estudio); T8: visita a las 8 semanas de tratamiento; T16: visita a las 16 semanas de tratamiento.

* significación estadística respecto al valor basal $p<0,05$. 


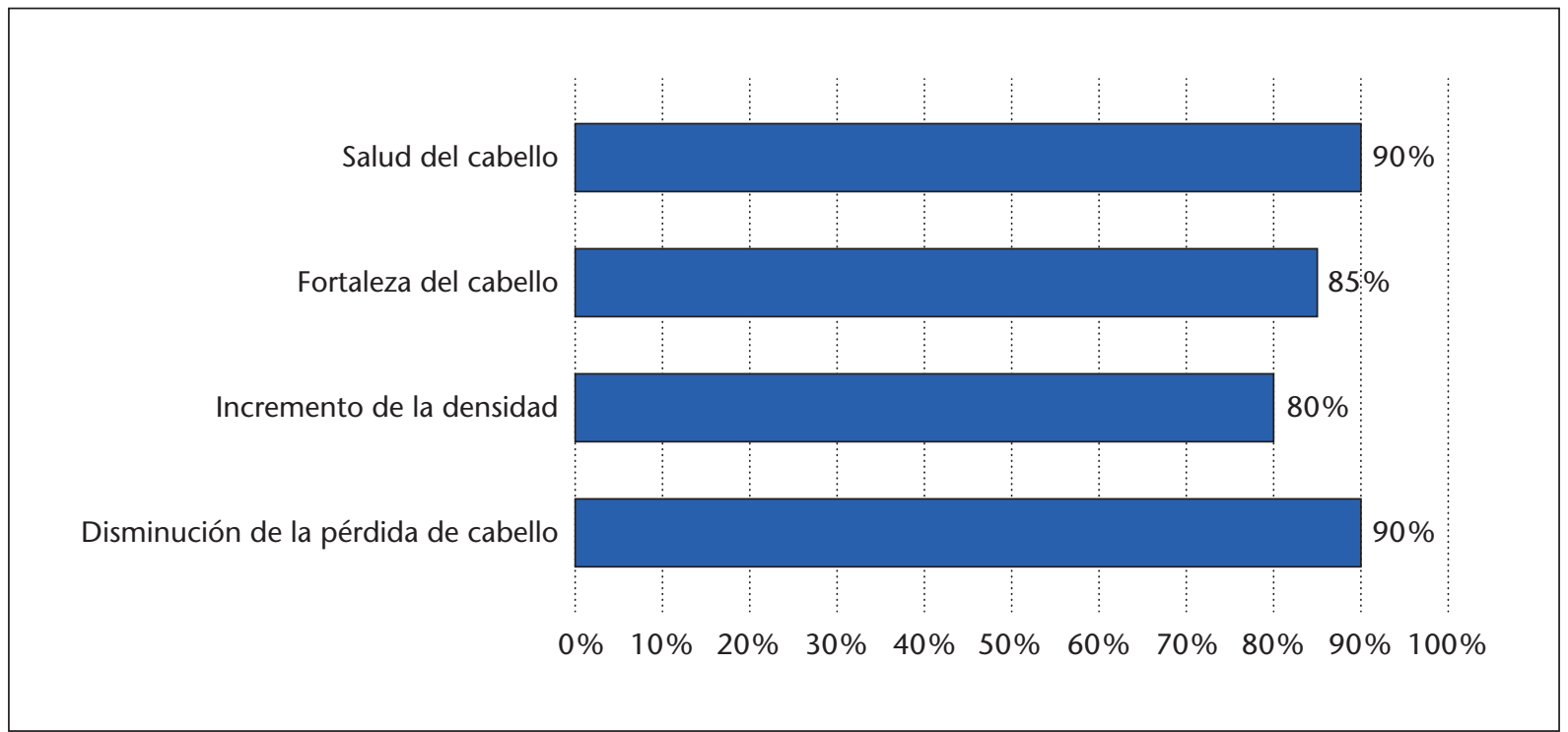

Figura 6. Evaluación subjetiva. Satisfacción. Porcentaje de pacientes que consideraron el tratamiento muy efectivo o bastante efectivo. Pygeum africanum-Serenoa repens en dosis de $100 \mathrm{mg}$ con MSM en dosis de $500 \mathrm{mg}$.

MSM: metilsulfonilmetano.

rados en humanos en las dosis recomendadas de uso $^{6}$.

Por otro lado, existe una relación directa entre la cantidad de extracto de Pygeum africanum + Serenoa repens y su actividad inhibitoria de la $5 \alpha-$ reductasa ${ }^{2}$. Gracias a esto, se puede considerar que existe margen de eficacia y de seguridad suficiente como para proponer un incremento de la dosis de los extractos Pygeum africanum/Serenoa repens, que cuenta con el aval de evidencia científica publicada, y conseguir un incremento de su actividad inhibitoria de la enzima $5 \alpha$-reductasa. «Mayor inhibición de la enzima $5 \alpha$-reductasa: mayor reducción del nivel androgénico» ${ }^{2}$.

En cuanto al MSM, se han demostrado importantes propiedades antioxidantes, gracias a su efecto potenciador de los niveles de tres antioxidantes naturales presentes en las células de la papila del folículo piloso: el glutatión, la superóxidodismutasa 1 y la catalasa. Un aumento en los niveles de estos antioxidantes durante el estrés oxidativo se va a traducir en un descenso de los marcadores de oxidación, como el malondialdehído, indicador de peroxidación lipídica² ${ }^{2}$ Así pues, gracias a estas propiedades, se ha planteado el uso de MSM en el tratamiento de la AAF, en busca de sus efectos beneficiosos antioxidantes. «Actividad antioxidante: alarga el ciclo de vida del cabello» ${ }^{2}$.

Además de sus propiedades antioxidantes, se ha demostrado que el MSM posee actividad antiinflamatoria. Concretamente, el tratamiento con MSM logra reducir los niveles de algunos mediadores inflamatorios liberados por los queratinocitos en las primeras etapas de la inflamación. Estos mediadores son: la interleucina $1 \alpha$, la interleucina $1 \beta$, el factor de necrosis tumoral, el factor de crecimiento transformante, la interleucina 6 y la interleucina $8^{2}$. Gracias a este efecto inhibidor, se ha planteado el uso de MSM en el tratamiento de la AAF, en busca de sus efectos beneficiosos antiinflamatorios, como tratamiento frente al factor inflamatorio identificado como uno de los factores implicados en la AAF. «Actividad antiinflamatoria: evita la fibrosis del folículo piloso» ${ }^{2}$.

Los resultados obtenidos en el presente estudio para Pygeum africanum/Serenoa repens en dosis de $100 \mathrm{mg}$ y MSM en dosis de $500 \mathrm{mg}$ indican que el aumento de la dosis de los extractos vegetales 
Pygeum africanum/Serenoa repens y la adición de MSM que proporciona un efecto antioxidante y antiinflamatorio, se traduce en una mejora de las variables de eficacia contempladas en el diseño del estudio con respecto a formulaciones anteriores con Pygeum africanum/Serenoa repens en dosis de $50 \mathrm{mg}$.

El análisis del fototricograma y la prueba de tracción demostraron que, tras el tratamiento durante 8 y 16 semanas con Pygeum africanum/Serenoa repens en dosis de $100 \mathrm{mg}$ con MSM en dosis de $500 \mathrm{mg}$, aumentó la proporción de cabellos en fase anágena (de crecimiento del cabello), a la vez que aumentaba la resistencia a la tracción del cabello. En comparación con la formulación con Pygeum africanum/Serenoa repens en dosis de $50 \mathrm{mg}$, el incremento en la proporción de cabellos en fase anágena fue un $30 \%$ mayor a las 16 semanas de tratamiento, y la mejora en la escala de la prueba de tracción, tres veces superior. De alguna manera, estos resultados ratifican la hipótesis de partida, en el sentido de lograr el objetivo de mejorar la efectividad del tratamiento con un abordaje clínico más integral.

Por otra parte, con el preparado de Pygeum africanum/Serenoa repens en dosis de $100 \mathrm{mg}$ con MSM en dosis de $500 \mathrm{mg}$, se logró una disminución significativa del eritema, así como del prurito, con una óptima tolerabilidad durante todo el tratamiento. Con estos resultados, por una parte, se confirma la seguridad avalada por los años de utilización en la práctica clínica de ambos extractos y que la OMS ya había descrito como bien tolerados en humanos y, por otra parte, se apunta al beneficio clínico añadido de haber incluido en la formulación el fecto antinflamatorio y antioxidante del MSM.

Finalmente, el estudio que aquí se presenta también encontró porcentajes de satisfacción elevados en la evaluación subjetiva realizada por las participantes tras 16 semanas de tratamiento con Pygeum africanum/Serenoa repens en dosis de 100 $\mathrm{mg}$ con MSM en dosis de $500 \mathrm{mg}$. Esto puede redundar en una mejora de la aceptación o la adherencia terapéutica que se requiere por parte de la paciente en este tipo de tratamiento, a largo plazo y sin descansos (intervalos de períodos sin tratamiento).

La principal innovación que ofrece la nueva formulación con el nombre comercial de Complidermol $\mathbf{5} \boldsymbol{\alpha}$ PLUS es un efecto adicional o un valor añadido en la efectividad del tratamiento de la AAF. Algo que podríamos llamar un «efecto PLUS» frente a formulaciones anteriores, como lo puede ser un PLUS en actividad y en beneficios para el tratamiento integral de la AAF:

- Aumentando la actividad inhibitoria de la enzima $5 \alpha$-reductasa.

- Reduciendo en mayor medida el paso de testosterona a dihidrotestosterona, y disminuyendo aún más los efectos androgénicos.

- Aumentando la actividad antioxidante, que reduce las especias reactivas del oxígeno.

- Reduciendo el estrés oxidativo, lo que alarga el ciclo de vida del cabello.

- Aumentando la actividad antiinflamatoria, que reduce la microinflamación local en el folículo piloso.

- Reduciendo la fibrosis y evitando la atrofia del folículo.

La nueva formulación resulta un complemento alimenticio que ofrece un abordaje clínico más integral de la AAF, aumentando su efecto sobre el conocido factor hormonal e incluyendo el tratamiento de los nuevos factores fisiopatológicos identificados (factor oxidativo e inflamatorio).

\section{CONCLUSIONES}

El mecanismo fisiopatológico por el cual tiene lugar la AAF es multifactorial y, en este sentido, además del factor genético y del hormonal, el factor oxidativo y la microinflamación aparecen cada vez con más fuerza como dianas imprescindibles sobre las que se debe intervenir para tratar la $\mathrm{AAF}$ con un abordaje clínico más integral. 
El preparado de Pygeum africanum en dosis de $100 \mathrm{mg} /$ Serenoa repens en dosis de $100 \mathrm{mg}$ con MSM en dosis de $500 \mathrm{mg}$ constituye un complemento alimenticio que vendría a contribuir a un abordaje clínico más integral de la AAF, aportando un valor añadido con respecto a formulaciones anteriores. Las principales aportaciones de la nueva formulación son: tratar el factor hormonal, incrementando la dosis de sus extractos vegetales de $50 \mathrm{mg}$ a $100 \mathrm{mg}$ cada uno de ellos, para aumentar la actividad inhibitoria de la enzima $5 \alpha$-reductasa; e incorporar una nueva molécula de MSM en dosis de $500 \mathrm{mg}$, con efecto antioxidante y antiinflamatorio demostrado para el tratamiento de los factores oxidativo e inflamatorio identificados como dianas de tratamiento.

En el análisis comparativo, se observa a las 16 semanas de tratamiento que, con la formulación de Pygeum africanum en dosis de $100 \mathrm{mg} /$ Serenoa repens en dosis de $100 \mathrm{mg}+\mathrm{MSM}$ en dosis de $500 \mathrm{mg}$, se obtiene con respecto a la formulación de Pygeum africanum en dosis de $50 \mathrm{mg} /$ Serenoa repens en dosis de $50 \mathrm{mg}$ : un aumento significativo del número de cabellos totales; un mayor aumento de la proporción de cabellos en fase anágena patente desde la visita T8, siendo un $30 \%$ superior en la visita de las 16 semanas; y una mejora de la resistencia del cabello a la tracción tres veces superior.

También se ha observado en el análisis comparativo que existe un mayor número de pacientes en quienes mejoran los parámetros de su AAF con respecto a su situación basal en el grupo de la nueva formulación. Así, vemos como el $100 \%$ de los casos mejoran en la proporción de cabellos en fase anágena tras 16 semanas de tratamiento frente a un $83 \%$ que lo hace en el grupo de la formulación anterior, y también que el $85 \%$ de los casos mejoran con la nueva formulación en la escala de resistencia a la tracción frente a un $61 \%$ que lo hace en el grupo de la formulación anterior.
$\mathrm{Al}$ igual que en la formulación anterior, la tolerabilidad fue buena, sin objetivarse ningún caso de efecto adverso que conllevara el abandono del tratamiento. En este sentido, los índices de satisfacción global obtenidos con una escala de valoración subjetiva alcanzaron porcentajes de satisfacción por encima del $80 \%$, entre los que cabe destacar la valoración de la salud de cabello y la disminución de la pérdida de cabello por encima del $90 \%$.

A la luz de los resultados obtenidos de este estudio comparativo frente a la formulación anterior de Pygeum africanum en dosis de $50 \mathrm{mg} /$ Serenoa repens en dosis de $50 \mathrm{mg}$, podríamos decir que, con las aportaciones de la nueva formulación, incrementando la dosis de sus extractos vegetales de $50 \mathrm{mg}$ a $100 \mathrm{mg}$ e incorporando la nueva molécula de MSM en dosis de $500 \mathrm{mg}$, con efecto antioxidante y antiinflamatorio, se objetiva un efecto adicional, «efecto PLUS», que mejora la efectividad del tratamiento y contribuye a un abordaje clínico más integral de la AAF.

\section{BIBLIOGRAFÍA}

1. Guerra-Tapia A, González-Guerra E, Borrás Schierloh JM. Alopecia androgénica femenina: nuevos factores fisiopatológicos y futuras tendencias para un abordaje clínico más integral. Más Dermatol. 2016;26:25-36.

2. Guerra-Tapia A, González-Guerra E, Borrás Schierloh JM. Alopecia androgénica femenina. Nuevas herramientas terapéuticas frente a los factores fisiopatológicos implicados: hormonal, oxidativo e inflamatorio. Más Dermatol. 2017;27: 21-33.

3. Wilt T, Ishani A, Stark G, Mac Donald R, Mulrow C, Lau J. Serenoa repens for benign prostatic hyperplasia. Cochrane Database Syst Rev. 2000;(2):CD001423.

4. Wilt T, Ishani A, Mac Donald R, Rutks I, Stark G. Pygeum africanum for benign prostatic hyperplasia. Cochrane Database Syst Rev. 2002;(1):CD001044.

5. Wilt TJ, Ishani A, Rutks I, Mac Donald R. Phytotherapy for benign prostatic hyperplasia. Public Health Nutr. 2000;3(4A): 459-72.

6. World Health Organization (WHO). WHO monographs on selected medicinal plants - volume 2. [Internet]. Ginebra: WHO; 2004. Disponible en: http://apps.who.int/medicinedocs/pdf/s4927e/s4927e.pdf 